ISSN-L 2077-0014

DOI: https://doi.org/10.33326/26176068.2021.3.1151

Artículo original/Original article/Artigo original

\title{
Violencia obstétrica en mujeres atendidas en un hospital público
}

\author{
Obstetric violence in women treated in a public hospital \\ Violência obstétrica em mulheres atendidas em um hospital público
}

Shirley Lizbeth Huarino-Suca ${ }^{1, a, b}$

Olga Choque-Chura ${ }^{1, a, c}$
D https://orcid.org/0000-0001-7038-1935

(iD) https://orcid.org/0000-0002-7604-0514

\section{Resumen}

Objetivo: Determinar la existencia de violencia obstétrica en mujeres con parto vaginal atendidas en el Hospital Hipólito Unanue de Tacna, Perú. Material y métodos: Estudio descriptivo, prospectivo, de corte transversal. Se aplicó el Cuestionario de Violencia Obstétrica (CVO) a 250 puérperas de 18 a 45 años de edad, de parto vaginal sin complicaciones y con recién nacido vivo y sano, de agosto a noviembre del 2019; los datos fueron procesados en el programa SPSS versión 23. Resultados: Las puérperas tuvieron entre 30 a 45 años (57,6 \%), estudios secundarios (74,4 \%), convivientes $(65,5 \%)$ y primíparas (67,5 \%). Durante la atención obstétrica, a veces o nunca recibieron información (34,8 \%), a veces o nunca pidieron consentimiento verbal para procedimientos (73,6 \%), a veces o nunca recibieron explicación sobre tacto vaginal $(34,8 \%)$, no recibieron explicación sobre ruptura de membranas $(48,8 \%)$, ni sobre episiotomía $(30,8 \%)$, ni sobre revisión uterina $(57,6 \%)$, a veces fueron llamadas por su nombre (32,8 \%), o tratadas con amabilidad y paciencia (73,2 \%), a veces con respeto y privacidad en la atención (63,2\%), no tuvieron acompañamiento durante el parto $(90,4 \%)$ y percibió maltrato verbal (17,6 \%). Presentaron ayuno $(65,6 \%)$, el parto fue en posición de litotomía $(77,2 \%)$, se practicó episiotomía (76,4 \%), contacto precoz a la hora (76 \%). Recibió maltrato (20,4 \%), siendo el 13,2 \% por personal técnico de enfermería. La violencia obstétrica fue por desinformación y falta de consentimiento (85,2 \%), trato deshumanizado (90,4%) y patologización o medicalización (99,6 \%). Conclusión: Existe violencia obstétrica en puérperas, al no permitir acompañamiento, no hay elección de posición de parto y se ejerce la episiotomía como rutina; se evidencia trato deshumanizado por personal de salud. Basta una sola forma de conducta, acción u omisión que afecte la atención de la gestante o puérpera para que se establezca la violencia obstétrica.

\section{Palabras clave: violencia, violencia obstétrica, personal de salud}

\begin{abstract}
Objective: To determine the existence of obstetric violence in women with vaginal delivery attended at the Hipólito Unanue Hospital in Tacna, Peru. Material and methods: Descriptive, prospective, cross-sectional study. The Obstetric Violence Questionnaire (CVO) was applied to 250 postpartum women aged 18 to 45, with uncomplicated vaginal delivery and live and healthy newborns, from August to November 2019; the data were processed in the SPSS version 23 program. Results: The puerperal women were between 30 and 45 years old (57.6 \%), secondary studies (74.4 $\%)$, partners (65.5\%) and primiparous women (67.5\%). During obstetric care, they sometimes or never received information (34.8\%), sometimes or never asked for verbal consent (73.6 \%), sometimes or never they received an explanation about the vaginal examination (34.8\%), they did not receive an explanation about rupture of membranes $(48.8 \%)$, or about episiotomy (30.8\%), or about uterine revision $(57.6 \%)$ ), sometimes they were called by name (32.8\%), or treated with kindness and patience $(73.2 \%)$, sometimes with respect and privacy in care $(63.2 \%)$, they were not accompanied during the delivery $(90.4 \%)$ and perceived verbal abuse (17.6\%). They were fasting $(65.6 \%)$, the delivery was in lithotomy position (77.2\%), episiotomy was performed $(76.4 \%)$, early contact at one hour (76\%). They received mistreatment $(20.4 \%)$, being $13.2 \%$ by technical nursing personnel. Obstetric violence was due to misinformation and lack of consent (85.2\%), dehumanized treatment (90.4\%) and pathologization or medicalization (99.6 \%). Conclusion: There is obstetric violence in puerperal women, by not allowing accompaniment, there is no choice of delivery position and episiotomy is performed as a routine; dehumanized treatment by health personnel is evidenced. A single form of conduct, action or omission that affects the care of the pregnant or puerperal woman is enough for obstetric violence to be established.
\end{abstract}

Keywords: violence, obstetric violence, health personnel

\footnotetext{
1 Universidad Nacional Jorge Basadre Grohmann. Tacna, Perú

a Licenciada en Obstetricia

${ }^{\mathrm{b}}$ Bachiller en Ciencias con mención en Obstetricia

c Doctor en Ciencias con mención en Salud Pública
} 


\begin{abstract}
Resumo
Objetivo: Determinar a existência de violência obstétrica em mulheres com parto vaginal atendidas no Hospital Hipólito Unanue de Tacna, Peru. Material e métodos: Estudo descritivo, prospectivo, de corte transversal. Foi aplicado o Questionário de Violência Obstétrica (CVO) a 250 puérperas de 18 a 45 anos de idade, de parto vaginal sem complicações e com recém-nascido vivo e saudável, de agosto a novembro de 2019; os dados foram processados no programa SPSS versão 23. Resultados: Os puérperas tiveram entre 30 e 45 anos (57,6 \%), estudos secundários (74,4 \%), conviventes (65,5 \%) e primíparas (67,5 \%). Durante os cuidados obstétricos, por vezes ou nunca receberam informação (34,8 $\%)$, pediram consentimento verbal para procedimentos, por vezes ou nunca $(73,6 \%)$, às vezes ou nunca receberam explicação sobre toque vaginal $(34,8 \%)$, não receberam explicação sobre ruptura de membranas $(48,8 \%)$, nem sobre episiotomia (30,8 \%), nem sobre revisão uterina (57,6 \%), às vezes foram chamadas pelo nome (32,8 \%), ou tratadas com amabilidade e paciência (73,2 \%), por vezes com respeito e privacidade no atendimento (63,2 \%), não tiveram acompanhamento durante o parto $(90,4 \%)$ e receberam abuso verbal (17,6 \%). Jejum (65,6 \%), parto na posição de litotomia $(77,2 \%)$, episiotomia $(76,4 \%)$, contato precoce horário (76 \%). Recebeu maus-tratos $(20,4 \%)$, sendo 13,2 \% por pessoal técnico de enfermagem. A violência obstétrica foi por desinformação e falta de consentimento (85,2 \%), tratamento desumanizado (90,4 \%) e patologização ou medicalização (99,6 \%). Conclusão: Existe violência obstétrica em puérperas, ao não permitir acompanhamento, não há escolha de posição de parto e se exerce a episiotomia como rotina; evidencia-se tratamento desumanizado por pessoal de saúde. Basta uma única forma de conduta, acção ou omissão que afecte a atenção da gestante ou puérpera para que se estabeleça a violência obstétrica.
\end{abstract}

Palavras-chave: violência, violência obstétrica, pessoal de saúde

\section{Introducción}

La Organización Mundial de la Salud (OMS) establece que la violencia obstétrica es una forma de violencia hacia la mujer, convirtiéndose en un problema de salud pública y una violación grave de los derechos humanos de las mujeres. ${ }^{1}$ En el Perú, durante el año 2019 , el $57,7 \%$ de mujeres fue víctima de violencia psicológica, física y sexual alguna vez en su vida, cifra menor en $5,5 \%$ en comparación con el año 2018, entre las formas de violencia destaca la violencia psicológica y/o verbal. $^{2}$ La violencia obstétrica ha sido afirmada por organismos internacionales y nacionales de derechos humanos, como una problemática que afecta principalmente a las mujeres por su condición de vulnerabilidad en el proceso de embarazo, parto y puerperio. Sin embargo, en el Perú es una de las manifestaciones de violencia menos reconocida. ${ }^{3,4}$

En España, un estudio menciona que de 17677 mujeres, el $34 \%$ opinó que había sufrido violencia obstétrica y que la atención obstétrica es deficitaria, desde el punto de vista científico y biomédico, pasando por encima del respeto a la dignidad, privacidad y a la autonomía de las mujeres. El $45,8 \%$ de las mujeres opinó que los sanitarios no solicitaron su consentimiento informado antes de cada técnica realizada y el $49 \%$ que no tuvo posibilidad de aclarar dudas o expresar miedos. El $38 \%$ percibió que durante el parto recibió procedimientos que no necesitaba y/o podían ser perjudiciales para su salud. ${ }^{5}$
En el Perú, en el Instituto Nacional Materno Perinatal se realizó un estudio que analizó la percepción de la paciente sobre la atención del parto humanizado, donde el $35 \%$ de las pacientes percibió que la atención del parto se realizó de forma adecuada, sin embargo, el $53 \%$ percibió que su atención fue a nivel regular y el $12 \%$ que fue inadecuado. ${ }^{6}$ Por otro lado, el Hospital Nacional Dos de Mayo es un establecimiento donde se realiza actividades docentes y se atiende pacientes obstétricas de estratos medio a bajo, donde el 75,4\% manifestó la realización de procedimientos médicos sin consentimiento informado, 69,4\% percibió trato deshumanizante, solo el $10,5 \%$ percibió trato no violento; la percepción de violencia obstétrica en esta institución es elevada y se relaciona a la realización de procedimientos sin consentimiento, falta de privacidad, maltrato del personal, realización de docencia. ${ }^{7}$ Asimismo, algunos estudios en las regiones de nuestro país reportan que existe violencia obstétrica en sus establecimientos de salud desde un $81,4 \%$ hasta el $100 \%{ }^{8-11}$

Es por ello que nos planteamos la siguiente interrogante: ¿Existe violencia obstétrica en puérperas de parto vaginal del Hospital Hipólito Unanue? En Tacna, no se han publicado estudios que aborden la violencia obstétrica como tal, en este contexto, conocer esta realidad permitirá asegurar la salud mental de las gestantes y puérperas, de ese modo disminuir el riesgo de desapego o depresión postparto, y por lo tanto generar un proceso gestacional saludable. De igual manera, buscar y 
proponer futuros cambios o estrategias sobre un tema tan importante, para diseñar y aplicar políticas públicas que disminuyan la tasa de violencia y mejorar las condiciones de atención a nivel nacional y regional.

El objetivo de este estudio fue determinar la existencia de violencia obstétrica en mujeres con parto vaginal atendidas en un hospital público de Tacna, Perú.

\section{Material y métodos}

Estudio descriptivo, prospectivo y de corte transversal en puérperas que han tenido su parto vaginal en el Hospital Hipólito Unanue de Tacna, entre los meses de agosto a noviembre del 2019. La muestra está constituida por 250 puérperas, seleccionadas por muestreo no probabilístico, que aceptaron participar en forma voluntaria del estudio, con edad entre 18 y 45 años, trabajo de parto sin complicaciones y recién nacido vivo y sano. El tamaño de muestra se determinó de acuerdo a la tabla de error de Fisher, Arkin y Colton, representativa para una población finita, con margen de error del $5 \%$ y con una confiabilidad del $95 \% \cdot 12,13$

Se tomó en consideración el Cuestionario de Violencia Obstétrica (CVO), construido por el Observatorio de Violencia Obstétrica de España, en el 2016, cuyo objetivo es evaluar la percepción y experiencia de violencia durante el parto, en sus aspectos desinformación, trato deshumanizado y medicalización y patologización. ${ }^{8}$ Este instrumento fue adaptado para la población peruana teniendo en cuenta las normas y protocolos para la atención del parto que existe en nuestro país. En la evaluación por expertos se decidió recolectar información detallada de la atención obstétrica, para después poder categorizarla en respuestas de Sí o No. Se mantuvieron los ítems ordenándolos en las tres dimensiones, con alto grado de acuerdo entre los expertos; y se realizó una prueba con 20 mujeres, semejante a la del estudio, para verificar el nivel de entendimiento de cada interrogante, finalmente el cuestionario respondía a nuestros objetivos y hacía posible una mejor descripción de la situación de violencia obstétrica en el Hospital Hipólito Unanue de Tacna.

El instrumento final quedó conformado por 6 interrogantes que recogen características sociodemográficas y 24 interrogantes de respuesta múltiple sobre violencia obstétrica que se categorizan en 19 items con respuestas dicotómicas, con valores de 0 y $1(0=$ No y 1 = Sí). El cuestionario está estructurado en tres dimensiones: desinformación (6 items), trato deshumanizado (5 items) y medicalización y patologización (8 items). El puntaje de cada dimensión se obtiene sumando el puntaje de las interrogantes de dicha dimensión y el puntaje global se obtiene al sumar los puntajes de las tres dimensiones, lo cual refiere mayores prácticas de violencia obstétrica.

Para el procesamiento de datos se trabajó con el programa SPSS Statistics versión 23 y Microsoft Office Excel 2013. Para interpretar los datos se utilizaron números absolutos y relativos. La presentación de los datos se hizo a través de tablas.

\section{Resultados}

En la Tabla 1 se puede observar que las pacientes atendidas, en su mayoría pertenecieron al grupo etario de 30 a 45 años con $57,6 \%$; mientras que un $42,4 \%$ de puérperas fueron gestantes con edades entre 18 y 29 años. Según el nivel de instrucción: la mayoría de la población entrevistada $(74,4 \%)$ terminó la secundaria y el $17,6 \%$ estudió superior universitario o no universitario. En cuanto al estado civil, el $65,6 \%$ es conviviente y el 17,2 $\%$, casada. En relación al lugar de procedencia, el $60,8 \%$ de las pacientes refieren proceder de alguna ciudad de la costa y el $86 \%$ de las pacientes refieren vivir en zona urbana. Finalmente, el $67,5 \%$ de puérperas dieron a luz por primera vez.

\section{Tabla 1}

Características sociodemográficas de puérperas atendidas de parto vaginal

\begin{tabular}{lcc}
\hline & $\mathrm{N}^{\circ}$ & $\%$ \\
\hline Edad & & \\
\hline De 18 a 29 años & 106 & 42,4 \\
De 30 a 45 años & 144 & 57,6 \\
\hline Nivel de instrucción & & \\
\hline Analfabeta & 2 & 0,8 \\
Primaria & 18 & 7,2 \\
Secundaria & 186 & 74,4 \\
Superior & 44 & 17,6 \\
\hline Estado civil & & \\
\hline Soltera & 37 & 14,8 \\
Conviviente & 164 & 65,6 \\
Casada & 43 & 17,2 \\
Divorciada & 6 & 2,4 \\
\hline Procedencia & & \\
\hline Costa & 152 & 60,8
\end{tabular}




\begin{tabular}{lcc} 
Sierra & 68 & 27,2 \\
Selva & 18 & 7,2 \\
Extranjero & 12 & 4,8 \\
\hline Residencia & & \\
\hline Urbano & 215 & 86 \\
Rural & 35 & 14 \\
\hline Paridad & & \\
\hline Primípara & 111 & 44,4 \\
Multípara & 139 & 55,6 \\
\hline
\end{tabular}

En la Tabla 2, respecto a la información sobre procedimientos durante su atención obstétrica, el $65,2 \%$ asegura haberla recibido siempre; el $27,2 \%$, solo a veces; y el 7,6 \%, nunca. El 60,8 $\%$ refiere que a veces se pidió su consentimiento para los procedimientos; un $26,4 \%$, siempre; y un $12,8 \%$, nunca. Por cuanto, el $65,2 \%$ siempre recibió explicación sobre el tacto vaginal; el 51,2\%, sobre la ruptura de membranas, el $69 \%$, sobre la episiotomía; y el 42,4\%, sobre la revisión uterina.

\section{Tabla 2}

Desinformación y falta de consentimiento en puérperas atendidas de parto vaginal

\begin{tabular}{lcc}
\hline & $\mathrm{N} .^{\circ}$ & $\%$ \\
\hline $\begin{array}{l}\text { Información sobre } \\
\text { procedimientos }\end{array}$ & & \\
\hline Siempre & 68 & 27,2 \\
A veces & 163 & 65,2 \\
Nunca & 19 & 7,6 \\
\hline
\end{tabular}

Consentimiento verbal informado

\begin{tabular}{lcc}
\hline Siempre & 66 & 26,4 \\
A veces & 152 & 60,8 \\
Nunca & 32 & 12,8 \\
\hline Explicación sobre el tacto & & \\
vaginal & & \\
\hline Siempre & 163 & 65,2 \\
A veces & 73 & 29,2 \\
Nunca & 14 & 5,6 \\
\hline Explicación sobre la ruptura & & \\
de membranas & & 51,2 \\
\hline Sí & 128 & 48,8 \\
No & 122 & \\
\hline Explicación sobre la & & \\
episiotomía & 173 & 69,2 \\
\hline Sí & 77 & 30,8 \\
No & & \\
\hline
\end{tabular}

\begin{tabular}{lll}
\hline $\begin{array}{l}\text { Explicación sobre la } \\
\text { revisión uterina }\end{array}$ & & \\
\hline Sí & 106 & 42,4 \\
No & 144 & 57,6 \\
\hline
\end{tabular}

En la Tabla 3 se describe tres indicadores del trato deshumanizado. La mayoría de las puérperas (64 \%) fue llamada por su nombre; el 32,8 \%, solo a veces; y un 3,2 \% fue llamada por diminutivos, número de cama u otros, pero no por su nombre. El $73,2 \%$ fue tratado con amabilidad y paciencia solo en ocasiones, $63,2 \%$ considera que solo a veces hubo respeto y privacidad en su atención, el mayor porcentaje $(90,4 \%)$ no tuvo acompañamiento durante el parto. Respecto al maltrato durante su atención obstétrica, el 79,6 \% no percibió ningún tipo de maltrato, el $17,6 \%$ fue víctima de maltrato verbal y el 2,8\% asegura haber sufrido maltrato físico.

\section{Tabla 3}

Trato deshumanizado en puérperas atendidas de parto vaginal

\begin{tabular}{lcc}
\hline & $\mathrm{N} .^{\circ}$ & $\%$ \\
\hline Fue llamada por su nombre & & \\
\hline Siempre & 160 & 64 \\
A veces & 82 & 32,8 \\
Nunca & 8 & 3,2 \\
\hline Trato con amabilidad y paciencia & & \\
\hline Siempre & 61 & 24,4 \\
A veces & 183 & 73,2 \\
Nunca & 6 & 2,4 \\
\hline Respeto y privacidad en la aten- & & \\
ción & & \\
\hline Siempre & 36 & 14,4 \\
A veces & 158 & 63,2 \\
Nunca & 56 & 22,4 \\
\hline Acompañamiento durante el & & \\
parto & & \\
\hline Sí & 24 & 9,6 \\
No & 226 & 90,4 \\
\hline Maltrato & & \\
\hline Físico & 7 & 2,8 \\
Verbal & 44 & 17,6 \\
Ninguno & 199 & 79,6 \\
\hline
\end{tabular}

En la Tabla 4 se aprecia que al $32 \%$ de las puérperas se les administró algún medicamento durante el parto, la mayoría permaneció en ayuno menos de 6 horas, al 51,6\% se le permitió la deambulación solo en ocasiones, el $77,2 \%$ de 
las puérperas refiere que dio a luz en la posición acostada con las piernas levantadas. Además, al $58,4 \%$ se le realizó la ruptura de membranas, al $76,4 \%$, episiotomía, el $24,8 \%$ refiere que ejercieron presión sobre su abdomen y a la mayoría (76 \%) se le permitió el contacto precoz con el recién nacido.

\section{Tabla 4}

Medicalización y patologización en puérperas atendidas de parto

\begin{tabular}{lcc}
\hline & $\mathrm{N} .^{\circ}$ & $\%$ \\
\hline $\begin{array}{l}\text { Uso de oxitocina u otro } \\
\text { medicamento }\end{array}$ & \\
\hline Sí & 80 & 32 \\
No & 164 & 65,6 \\
No lo sabe & 6 & 2,4 \\
\hline Dieta & & \\
\hline Dieta blanda & 6 & 2,4 \\
Dieta líquida & 80 & 32 \\
Ayuno menos de 6 horas & 106 & 42,4 \\
Ayuno más de 6 horas & 58 & 23,2 \\
\hline Deambulación & & \\
\hline Siempre & 45 & 18 \\
A veces & 129 & 51,6 \\
Nunca & 76 & 30,4 \\
\hline
\end{tabular}

Posición

\begin{tabular}{lcc}
\hline Acostada y con las piernas & 193 & 77,2 \\
levantadas & 56 & 22,4 \\
Semisentada & 1 & 0,4 \\
En cuatro puntos & & \\
\hline Ruptura de membranas & 146 & 58,4 \\
\hline Artificial & 104 & 23,6 \\
Espontánea & & \\
\hline Episiotomía & 191 & 76,4 \\
\hline Sí & 59 & 41,6 \\
No & & \\
\hline Maniobra de Kristeller & 62 & 24,8 \\
\hline Sí & 188 & 75,2 \\
No & & \\
\hline Permitir contacto precoz & 190 & 76 \\
\hline Sí, durante una hora & 60 & 24 \\
Sí, menos de una hora & & \\
\hline
\end{tabular}

En la Tabla 5 tenemos los datos que señalan que quienes ejercían algún tipo de violencia fueron los médicos, las obstetras y el personal técnico de enfermería, en 4,8 \%, 2,4 \% y $13,2 \%$ respectivamente. Según estas cifras, la mayoría percibió algún tipo de violencia por parte del personal técnico, lo que se constituye como un factor de riesgo a violencia obstétrica.

Tabla 5

Personal de salud involucrado con maltrato en puérperas atendidas de parto vaginal

\begin{tabular}{lcc}
\hline & $\mathrm{N} \cdot{ }^{\circ}$ & $\%$ \\
\hline Médico & 12 & 4,8 \\
Obstetra & 6 & 2,4 \\
Técnico de enfermería & 33 & 13,2 \\
Nadie & 199 & 79,6 \\
\hline Total & 250 & 100 \\
\hline
\end{tabular}

En la Tabla 6, de acuerdo a los datos procesados, existe desinformación y falta de consentimiento en el $85,2 \%$, trato deshumanizado en el 90,4 \% y patologización y medicalización en el 99,6 $\%$. Entonces, se puede decir que la violencia obstétrica en general es percibida por el total de la muestra de estudio.

\section{Tabla 6}

Dimensiones de la violencia obstétrica en puérperas atendidas de parto vaginal

\begin{tabular}{ccccccc}
\hline \multirow{2}{*}{$\begin{array}{c}\text { Violencia } \\
\text { obstétrica }\end{array}$} & $\begin{array}{c}\text { Desinformación } \\
\text { y falta de } \\
\text { consentimiento }\end{array}$ & \multicolumn{2}{c}{$\begin{array}{c}\text { Trato } \\
\text { deshumanizado }\end{array}$} & $\begin{array}{c}\text { Patologización y } \\
\text { medicalización }\end{array}$ \\
\cline { 2 - 7 } Sí & 213 & 85,2 & 226 & 90,4 & 249 & 99,6 \\
No & 37 & 14,8 & 24 & 9,6 & 1 & 0,4 \\
\hline Total & 250 & 100 & 250 & 100 & 250 & 100 \\
\hline
\end{tabular}

\section{Discusión}

De acuerdo a los resultados obtenidos, se puede decir que la violencia obstétrica ha sido ejercida en nuestra muestra de estudio; es decir, ellas percibieron por lo menos una forma de conducta, acción u omisión de parte de los profesionales de salud que afectó la atención obstétrica recibida. En el hospital de estudio la responsabilidad del alto porcentaje de violencia obstétrica en puérperas recae en los profesionales de salud, al brindar una atención sin tomar en cuenta el contexto sociodemográfico y cultural, enfocándose solamente en el cuidado biológico. Estos resultados son coincidentes con Cerpa y Velásquez quienes reportan que existe una elevada percepción de violencia obstétrica en el 98,4 $\%$ de pacientes. ${ }^{10}$ 
En relación a las dimensiones de la violencia obstétrica, podemos observar que, del total de puérperas, al $7,6 \%$ no se le brindó información, así como no se le solicitó su consentimiento al 12,8 $\%$. Es decir, se les niega el derecho de la toma de decisión informada, dando a las parturientas información insuficiente, ninguna información o desinformando acerca de sus opciones, impidiendo el involucramiento de la madre en la toma de decisiones. ${ }^{14}$ Estos datos difieren en porcentaje con los encontrados por Iglesias et al. donde el 45,8 $\%$ de las mujeres opinaron que los sanitarios no solicitaron su consentimiento informado antes de cada técnica realizada. ${ }^{5}$ Asimismo, en Moreno, el 75,4 \% manifestó la realización de procedimientos médicos sin consentimiento informado. ${ }^{7}$

En el Hospital Hipólito Unanue, una vez hospitalizada la gestante se convierte en "paciente", esto quiero decir que es objeto del acto médico, por lo que debe obedecer todo lo indicado por el profesional de salud, quien posee y a quien se le reconoce el conocimiento amplio de la situación, específicamente del proceso gestacional. Algo también observado fue el poco involucramiento de la pareja o familia en la toma de decisiones, en algunas ocasiones los procedimientos eran decisión exclusiva del médico de turno, olvidando que la mujer tiene el derecho de ser informada sobre su atención obstétrica.

Por otro lado, se puede observar que el 90,4 $\%$ no tuvo acompañamiento de algún familiar durante el parto, considerándose así que han recibido un trato deshumanizado por esta prohibición, en comparación con el estudio de Paucar, se muestra similitud señalando que al $92,5 \%$ se les impidió el acompañamiento. ${ }^{9}$ En nuestro estudio, respecto al personal de salud involucrado en maltrato, el 13,2 $\%$ señaló a la técnico de enfermería como causante; el 4,8 \%, al médico; y el 2,4\%, a la obstetra. En nuestra opinión, algunos profesionales de la salud tienen una conducta de poder y ejercen un trato indiferente contra la mujer parturienta, en momentos tan críticos como el trabajo de parto no se da una atención de calidez, esto fue evidenciado también en la investigación de Orcotorio al señalar una mala interrelación entre el personal de salud y el paciente en el $74,6 \%$ y al personal técnico en enfermería como un factor de riesgo para violencia obstétrica. ${ }^{11} \mathrm{El}$ parto y el puerperio son momentos claves por los cambios que experimenta física y emocionalmente la mujer, lo que la posiciona en un estado de vulnerabilidad y el trato hacia ella debería ser humanista.
En la dimensión medicalización y patologización, observamos que el $77,2 \%$ dio a luz en posición de litotomía, sin la opción de poder elegir una posición más cómoda, así como al $30,4 \%$ no se les permitió caminar o cambiar de posición mientras transcurrían las horas esperando completar el periodo de dilatación, resultados muy similares a los hallados en el estudio de Vela donde el $75 \%$ de las pacientes manifestaron que nunca se les explicó que podían escoger la posición de su parto y al $79 \%$ nunca le enseñaron posiciones de parto. ${ }^{6}$ Lo anterior se contradice a las recomendaciones de la OMS: "Se recomienda alentar la movilidad y adoptar una posición erguida durante el trabajo de parto en mujeres de bajo riesgo" y "Para las mujeres sin anestesia peridural, se recomienda alentar la adopción de una posición para dar a luz que sea de su elección, inclusive las posiciones erguidas". ${ }^{15}$ En ocasiones se observó que eran sometidas a monitoreo estricto sin tener factor de riesgo, con el pasar de las horas se agudizaba el dolor producto de esa posición incómoda; luego en el periodo expulsivo ya no tenían fuerzas para sostenerse por sí solas y mucho menos para pujar, lo que conducía a situaciones complicadas, ya que sin el pujo adecuado el periodo expulsivo solía extenderse y el personal a cargo terminaba realizando episiotomía o maniobra de Kristeller, situación que se puede evitar si se aplica la Norma Técnica de Salud para la Atención del Parto Vertical en el marco de los Derechos Humanos con Pertinencia Intercultural, permitiendo la libre deambulación en mujeres sin factores de riesgo y con trabajo de parto espontáneo. ${ }^{16}$

Por todo lo dicho, la violencia obstétrica merece un abordaje mayor en su totalidad, debe ser diferenciada y caracterizada. Según los resultados las causas podrían ser la dinámica de las relaciones de poder desiguales entre el personal de salud y la gestante o puérpera, la inadecuada formación del personal de salud, no del conocimiento científico, sino de la formación de empatía con el paciente para reconocer las necesidades y otros enfoques a tomar en consideración durante la atención a una mujer que experimenta muchos cambios físicos y emocionales.

En nuestro país no existe una legislación contra la violencia obstétrica, se han presentado dos proyectos de ley relacionados al parto humanizado, pero fueron archivados. Contamos con el Observatorio Nacional de la violencia contra las mujeres y los integrantes del grupo familiar, pero este no contiene estadísticas sobre violencia obstétrica. ${ }^{17}$ No se cuenta con protocolos ni normas específicas para su intervención en la prevención, 
sanción y erradicación; si bien existe el Plan Nacional contra la violencia de género con vigencia hasta el presente año, este no aborda la violencia obstétrica y solo la conceptualiza como un tipo más de violencia hacia la mujer. ${ }^{18}$ Por ello, y como primer paso, es necesario realizar estudios como este, para la profundización sobre las dimensiones, factores, causas y particularidades relacionadas a la violencia obstétrica, y usarlos como base para en un futuro desarrollar e implementar normativas específicas sobre este problema de salud pública.

\section{Conclusión}

Existe violencia obstétrica en mujeres con parto vaginal atendidas en el Hospital Hipólito Unanue de Tacna, durante la atención existió al menos una forma de conducta, acción u omisión por parte del personal de salud que afectó de alguna manera el proceso de parto.

\section{Referencias}

1. Organización Mundial de la Salud. Prevención y erradicación de la falta de respeto y el maltrato durante la atención del parto en centros de salud. Declar la OMS [Internet]. 2014;1-4. Disponible en: https://apps.who.int/iris/bitstream/ handle/10665/134590/WHO_RHR_14.23_spa. pdf;jsessionid=657F79222E756730592D601CEE39C812? sequence $=1$

2. Instituto Nacional de Estadística e Informática. Encuesta Demográfica y de Salud Familiar-ENDES [Internet]. 2015 [citado 28 de marzo de 2021]. Disponible en: www.inei.gob.pe

3. Quattrocchi P. Violencia Obstétrica. Aportes Desde América Latina. Gênero \& Direito [Internet]. 2018;7(1):20-46. Disponible en: https://periodicos.ufpb.br/index.php/ged/article/ view/38974/19649

4. Díaz García LI, Fernández M. Y. Situación legislativa de la Violencia obstétrica en América latina: el caso de Venezuela, Argentina, México y Chile. Rev derecho [Internet]. 2018 [citado 28 de marzo de 2021];(ahead):0-0. Disponible en: https://scielo.conicyt.cl/scielo.php?script=sci_arttext\&pid=S0718-68512018000200123\&Ing=es\&nrm=iso\&tlng=es

5. Iglesias S, Conde M, González S, Parada ME. ¿Violencia obstétrica en España, reali- dad o mito? 17.000 mujeres opinan. MUSAS Rev Investig en Mujer, Salud y Soc [Internet]. 31 de enero de 2019 [citado 21 de marzo de 2021];4(1):77-97. Disponible en: https://revistes.ub.edu/index.php/MUSAS/article/view/vol4. num1.5

6. Vela Coral G del P. Percepción de la paciente sobre la atención del parto humanizado en el Servicio de Centro Obstétrico del Instituto Nacional Materno Perinatal durante el periodo marzo-abril de 2015. 2015 [citado 29 de marzo de 2021]; Disponible en: https://cybertesis.unmsm.edu.pe/handle/20.500.12672/4278

7. Moreno-Garrido ZR. Percepción de violencia obstétrica en pacientes atendidas en un Hospital Docente de Lima Perú. 2018. Rev Int Salud Matern Fetal [Internet]. 20 de noviembre de 2019 [citado 28 de marzo de 2021];4:S14-S14. Disponible en: http://ojs.revistamaternofetal. com/index.php/RISMF/article/view/125

8. Lam Flores SL, Sanchez Romero VJ, Alva Olivos MA, Castillo Vásquez JS, Cabellos Tufinio P. Violencia obstétrica y estrés en puérperas atendidas en un hospital de Chimbote, 2017 [Internet]. Universidad San Pedro. Universidad San Pedro; 2017 feb [citado 28 de marzo de 2021]. Disponible en: http://repositorio.usanpedro.edu. pe/handle/USANPEDRO/314

9. Paucar Briones R del C. Violencia obstétrica percepción de las gestantes en trabajo de parto Hospital Regional Docente de Cajamarca 2017. 2018 [citado 29 de marzo de 2021]; Disponible en: http://repositorio.unc.edu.pe/handle/ UNC/1801

10. Cerpa Rodríguez CD, Velásquez Chauca M del C. Violencia obstétrica en mujeres atendidas de parto eutócico. Servicio de Gineco-obstetricia. Hospital Regional Honorio Delgado Espinoza. Abril - Junio 2015 [Internet]. 2015 [citado 21 de marzo de 2021]. Disponible en: http:// tesis.ucsm.edu.pe/repositorio/bitstream/handle/ UCSM/3390/63.0656.OP.pdf?sequence $=1$ \&isAllowed=y

11. Orcotorio Quispe YO. Factores asociados a violencia obstétrica durante el parto en mujeres atendidas en el servicio de obstetricia del Hospital Regional del Cusco, 2019 [Internet]. Universidad Andina del Cusco. Universidad Andina del Cusco; 2019 [citado 28 de marzo de 2021]. 
Disponible en: http://repositorio.uandina.edu. pe:8080/xmlui/handle/UAC/2560

12. Hernández Sampieri R, Fernández Collado $C$, Baptista Lucio M del P. Metodología de la Investigación [Internet]. 6ta ed. Rocha Martínez MI, editor. México; 2014 [citado 21 de marzo de 2021]. Disponible en: https://www.uca.ac.cr/ wp-content/uploads/2017/10/Investigacion.pdf

13. Zevallos Ypanaqué G, Palomino Orizano JA, Peña Corahua JD, Orizano Quedo LA. Metodología de la investigación [Internet]. [citado 28 de marzo de 2021]. Disponible en: https://isbn. cloud/9786123152628/metodologia-de-la-investigacion/

14. Arguedas Ramírez G. La violencia obstétrica: propuesta conceptual a partir de la experiencia costarricense. Cuad Inter.cambio sobre Centroamérica y el Caribe [Internet]. 2014 [citado 27 de marzo de 2021];11(1):145-69. Disponible en: http://www.redalyc.org/articulo. oa?id=476947241008

15. Organización Mundial de la Salud. Recomendaciones de la OMS para los cuidados durante el parto, para una experiencia de parto positiva. WHO [Internet]. 2019 [citado 28 de marzo de
2021]; Disponible en: http://www.who.int/reproductivehealth/publications/intrapartum-care-guidelines/es/

16. Ministerio de Salud. Norma Técnica de Salud para la Atención del Parto Vertical en el marco de los Derechos Humanos con Pertinencia Intercultural [Internet]. 2016 [citado 28 de marzo de 2021]. Disponible en: http://bvs.minsa.gob. pe/local/MINSA/4240.pdf

17. Ministerio de la mujer y poblaciones vulnerbles. Observatorio Nacional de la Violencia contra las Mujeres y los Integrantes del Grupo Familiar [Internet]. 2020 [citado 21 de marzo de 2021]. Disponible en: https://observatorioviolencia.pe/ mv_violencia-obstetrica/

18. Ministerio de la mujer y poblaciones vulnerables. Decreto Supremo que aprueba el Plan Nacional Contra la Violencia de Género 2016 - 2021 [Internet]. 2016 [citado 27 de marzo de 2021]. Disponible en: https://www.mimp.gob.pe/ empresasegura/decreto-supremo-que-aprueba-el-plan-nacional-contra-la-viol-DS-0082016-mimp.pdf

\section{Correspondencia:}

shirley.huarino@unjbg.edu.pe
Fecha de recepción: 28/05/2021

Fecha de aceptación: 05/08/2021 